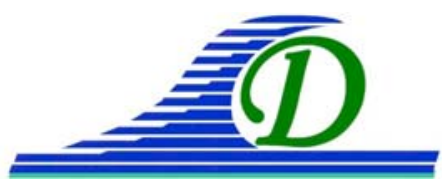
XIII ${ }^{\text {èmes }}$ Journées Nationales Génie Côtier - Génie Civil
Dunkerque, 2-4 juillet 2014

DOI:10.5150/jngcgc.2014.103 @ Editions Paralia CFL

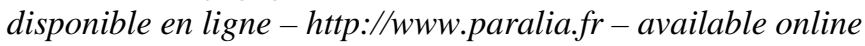

\title{
Mise en perspective de la vulnérabilité des enjeux à l'érosion et aux différents types d'inondation sur la partie sableuse du Languedoc-Roussillon
}

\section{Paul SAUBOUA ${ }^{1}$, Hugues HEURTEFEUX ${ }^{2}$}

\section{EID Méditerranée, Pôle littoral,}

165 avenue Paul Rimbaud, F-34184 Montpellier Cedex 4, France.

psauboua@eid-med.org

2. EID Méditerranée, Pôle littoral,

165 avenue Paul Rimbaud, F-34184 Montpellier Cedex 4, France.

hheurtefeux@eid-med.org

\section{Résumé :}

Cette étude a permis d'évaluer la vulnérabilité des enjeux à l'érosion, la submersion marine et aux autres types d'inondation sur le littoral du Languedoc-Roussillon. Réalisée en 2013, elle a mobilisé les résultats de travaux effectués dans le cadre du CPER 2007-2013 par l’EID Méditerranée et le BRGM ainsi que des données produites par les services de l'Etat, notamment de la DREAL LR.

L'évaluation de la vulnérabilité des enjeux sur le littoral s'est faite en croisant zonages d'aléas et enjeux géoréférencés par traitement sous logiciel de SIG ArcGis v¹0.0. Dans cette étude, ce sont plusieurs aléas présents sur le littoral qui ont été étudiés au travers d'une analyse multicritères, dans laquelle des critères quantitatifs ont été précisés au moyen de critères qualitatifs. Les zonages de vulnérabilité ont été croisés à 18 catégories d'enjeux réparties en trois thématiques : santé humaine, économie, lié à l'environnement. Le long des $190 \mathrm{~km}$ de la côte sableuse du Languedoc-Roussillon, ce sont près de 94000 bâtiments et $3641 \mathrm{~km}$ de réseaux qui ont été étudiés. La représentation des résultats s'est faite au moyen d'un atlas dans lequel le linéaire étudié a été subdivisé en 38 secteurs. Pour chacun de ces secteurs, la vulnérabilité à l'érosion et à la submersion marine et aux autres types d'inondation ont fait l'objet de deux cartes employables au $1: 25000$. Ces cartes ont été associées à des histogrammes donnant pour chacune des 18 catégories d'enjeux son importance vis-à-vis du reste du littoral et sa valeur de vulnérabilité globale.

Mots-clés : Vulnérabilité, Erosion, Submersion marine, Analyse multi-critères, Languedoc-Roussillon.

\section{Introduction}

Les dernières orientations stratégiques de gestion du littoral en Languedoc-Roussillon datant de la Mission Interministérielle d'Aménagement du Littoral en 2003, il paraissait nécessaire dans le cadre du Contrat de Projet Etat-Région (CPER) 2007-2013 de 


\section{Thème 7 - Risques côtiers}

proposer des orientations de gestion actualisées. Ce travail avait donc pour objectif de définir la vulnérabilité des enjeux présents sur ce littoral au regard des aléas érosion et submersion marine présents à l'horizon 2030, ainsi que des autres types d'inondation.

L'étude s'est portée sur les communes littorales ayant une façade maritime entre le Racou (Pyrénées Orientales) et le Grau-du-Roi (Gard), correspondant à un linéaire côtier sableux de près de 190 kilomètres. A partir de la définition de la vulnérabilité du territoire (ii), la méthode employée a été basée sur la caractérisation précise des enjeux présents (iii). Le croisement de ces données (iv) a permis l'obtention de résultats (v) représentés sous forme de cartes et d'histogrammes, donnant pour chaque enjeu sa valeur de vulnérabilité globale et son importance vis-à-vis du reste du littoral.

\section{Définition de la vulnérabilité du territoire aux aléas érosion, submersion marine et aux autres types d'inondation}

\subsection{Méthode d’évaluation de la vulnérabilité}

\subsubsection{Recours à une analyse multi-critères}

La vulnérabilité exprime et mesure le niveau de conséquences prévisibles de l'aléa sur les enjeux (MEDDE, 2009). Cette étude a été basée sur une analyse multi-critères afin de préciser les valeurs de vulnérabilité aux aléas érosion et submersion marine obtenus depuis des travaux du BRGM et de la SOGREAH effectués dans le cadre du CPER en 2011. Il a été décidé d'employer des facteurs additionnels à ces résultats afin de rendre cette étude plus complète.

Pour l'érosion, il s'agit de données relatives à l'existence d'aménagements de protection douce sur le littoral. De la même façon, au-delà de la vulnérabilité à la submersion, c'est la question de la vulnérabilité aux inondations qui doit être considérée dans une perspective de préconisations de gestion. Or en zones littorales basses, qui composent l'essentiel du littoral du Languedoc-Roussillon, d'autres aléas peuvent entraîner des inondations. Les crues, les élévations du niveau des lagunes retro-littorales et les remontées de nappes sont autant de facteurs pouvant générer des inondations. Ils se produisent d'ailleurs souvent de manière concomitante avec les submersions marines.

\subsubsection{Eléments de cadrage}

Il a été décidé de présenter la vulnérabilité du littoral du Languedoc-Roussillon, côtes essentiellement basses et sableuses avec des lidos, à l'horizon 2030. Ainsi, l'échelle temporelle est celle de la préparation à l'action. Concernant l'échelle de réflexion, la sectorisation en 38 unités hydrosédimentaires du Racou (Pyrénées Orientales) au Graudu-Roi (Gard) de BRUNEL (2013) a été employée. 


\section{XIII ${ }^{\text {èmes }}$ Journées Nationales Génie Côtier - Génie Civil \\ Dunkerque, 2-4 juillet 2014}

\subsection{Vulnérabilité à l'érosion}

La note globale de vulnérabilité à l'érosion a été déterminée par la valeur de vulnérabilité à la distance au trait de côte, de laquelle est déduite une valeur méliorative d'aménagement de protection douce :

Vuln. à l'érosion $=$ Vuln. dist. au trait de côte - Val d'amgt. de protection douce

\subsubsection{Critère quantitatif : distance au trait de côte}

Cette étude s'est appuyée sur les résultats du travail de la SOGREAH (2011) relatif à l'actualisation de l'aléa érosion en Languedoc-Roussillon dans le cadre du CPER. A partir de ce trait de côte supposé, cinq classes ont été définies par bandes de 25 et $50 \mathrm{~m}$, et une valeur de vulnérabilité a été attribuée en fonction de l'éloignement au trait de côte. La classification a été basée sur la valeur des 100 mètres, qui d'après le paragraphe III de l'article L 146-4 de loi n86-2 du 3 janvier 1986 relative à l’aménagement, la protection et la mise en valeur du littoral, peut être considérée comme la distance en deçà de laquelle les enjeux présentent une vulnérabilité à l'érosion.

\subsubsection{Critères qualitatifs : présence et état d'aménagements de protection douce}

Une action du CPER avait permis en 2010 d'obtenir une évaluation des aménagements de protection douce sur le littoral du Languedoc-Roussillon (EID, 2011). 215 aménagements, apports sableux et maillages de ganivelles, participant à la gestion morphologique du littoral y avaient été étudiés. L'existence de tels aménagements de protection douce sur un secteur permet à celui-ci de mieux appréhender le recul, influant à partir d'une certaine largeur de plage sur la vitesse de son recul. Ce paramètre a donc été pris en compte dans l'attribution de la valeur de vulnérabilité à l'érosion.

La présence de ces ouvrages a permis d'affecter une valeur méliorative à la valeur de vulnérabilité attribuée aux enjeux vis-à-vis de leur distance au trait de côte. Cette valeur méliorative dépendait de la nature du ou des aménagements présents, ainsi que de leur état et efficacité, et pouvait diminuer jusqu'à près d'un quart la valeur de vulnérabilité.

\subsection{Vulnérabilité à la submersion et aux autres types d'inondation}

2.3.1 Critères employés, définition d'une note globale de vulnérabilité aux différents types d'inondation

Dans une perspective de préconisations de gestion, la réflexion sur la vulnérabilité aux aléas littoraux ne peut être limitée à l’aléa submersion marine, ni à la frange littorale. Le contexte géomorphologique du Languedoc-Roussillon se traduit par une vulnérabilité des enjeux littoraux à la submersion marine, mais aussi aux crues, aux élévations du niveau des lagunes et aux remontées de nappes. Ces aléas ont donc aussi été pris en compte dans cette étude. En outre, les zones retro-littorales peuvent aussi être impactées par ces aléas. 


\section{Thème 7 - Risques côtiers}

Pour la zone littorale, la note globale de vulnérabilité aux différents types d'inondation a été obtenue à partir de l'évaluation de plusieurs critères :

- Les hauteurs d'eau et périodes de retour des submersions. Ce critère a été précisé au moyen d'un facteur historique des tempêtes, fonction du nombre d'évènements météo-marins qu’a subi le secteur, obtenu à partir des données CatNat depuis 1982.

- La localisation des zones potentiellement inondables par crue de l'atlas des zones inondables (DREAL LR, 2010), précisé par un facteur historique des tempêtes.

- La localisation en zone sur laquelle des inondations par élévations du niveau des lagunes sont possibles, à partir de l'atlas des zones inondables par submersion marine (DREAL LR, 2009).

- La localisation en zone sur laquelle des inondations par remontée de nappes avaient déjà été observées à partir d'une étude encadré par le Service Maritime et de Navigation du Languedoc-Roussillon (LAPEYRE, 2004).

En zone retro-littorale la note globale de vulnérabilité aux différents types d'inondation a été obtenue à partir de l'évaluation des mêmes critères, excepté pour la submersion marine. Ne disposant pas de données LIDAR pour ces secteurs, l'évaluation s'est faite à partir du recensement des zones inondées par submersion marine (LAPEYRE, 2004).

2.3.2 Description des critères quantitatifs : hauteurs d'eau et période de retour des submersions

La plupart des études d'évaluation de la vulnérabilité à la submersion marine ne prennent en compte que l'aléa de référence, i.e. l'aléa de période de retour centennale. Or la probabilité d'occurrence est un paramètre aussi important à la définition de l'aléa que son intensité. Les dommages causés par des évènements de période de retour plus faible ne sont pas pris en compte alors que leur fréquence étant plus élevée, si les dommages induits sont importants il semble nécessaire de considérer leur influence.

Pour ces raisons, l'aléa submersion a été étudié aux périodes de retour annuelle, décennale, cinquantennale et centennale. Pour les trois premières périodes de retour, les travaux réalisés se sont appuyés sur les résultats du BRGM (2011). Le zonage pour la période de retour centennale a été effectué dans cette étude en se basant sur les préconisations de la DREAL LR (2012) et à partir de données LIDAR de 2009.

Pour chaque surface de territoire, en fonction de son altitude, le travail préalable aura permis par traitement sous logiciel de SIG ArcGis v¹0.0 d’y attribuer une valeur de vulnérabilité à la submersion marine, pour chacune des périodes de retour prises en compte. La valeur de vulnérabilité globale a ensuite été construite à partir de ces différentes valeurs et de leurs probabilités d'occurrence respectives. Elle correspond dans ce sens à la vulnérabilité probable pour une année.

Cette valeur de vulnérabilité globale à la submersion marine a ensuite été précisée par l’historique des tempêtes et les valeurs de vulnérabilité aux autres aléas inondation. 


\section{XIII ${ }^{\text {èmes }}$ Journées Nationales Génie Côtier - Génie Civil \\ Dunkerque, 2-4 juillet 2014}

\section{Définition des enjeux présents sur le littoral du Languedoc-Roussillon}

\subsection{Identification des catégories d'enjeux}

Par cohérence entre les documents d'orientations de gestion, les catégories d'enjeux utilisées ont été les mêmes que celles préconisées par la directive européenne sur l'évaluation et la gestion des risques d'inondation, et celles employées pour l'évaluation préliminaire des risques inondations. Le patrimoine culturel, les zones Natura 2000 et ZNIEFF n'ont pas été traités. Les catégories d'enjeux étudiés appartiennent donc à trois différentes thématiques: les enjeux de santé humaine, les enjeux économiques et les enjeux liés à l'environnement.

Les données géoréférencées sur les enjeux ont été obtenues à partir de bases de données de l'IGN, de l'INSEE et de services de l'Etat. La définition des enjeux a été réalisée en grande partie au moyen de la BD-TOPO ${ }^{\circledR}$ V2.1 de l'IGN (2011). Les données de l'INSEE ont permis de préciser des éléments relatifs à la population et aux activités économiques (INSEE, 2009 \& 2011) et celles des services de l'Etat les enjeux liés à l'environnement (DREAL LR, 2011 ; MEDDE, 2012).

\subsection{Enjeux de santé humaine}

La vulnérabilité des personnes a été évaluée au travers de la population présente sur le territoire, ainsi que du nombre de bâtiments d’hébergement, d'accueil et d'appui à la population. Huit indicateurs ont ainsi été étudiés :

- Population : Population habitant sur un secteur considéré comme vulnérable ;

- Population vivant dans des habitations de plain-pied : Population sans espace refuge ;

- Habitations : Nombre (Nb) d’habitations en secteur vulnérable ;

- Habitations de plain-pied : Nb d'habitations sans espace refuge ;

- Hébergements touristiques : Nb de campings et villages de vacances ;

- Etablissements publics administratifs et d'appui à la population : Nb de bâtiments

- Etablissements publics d'enseignement: $\mathrm{Nb}$ d'établissements Iaire, IIaire et supérieurs ;

- Etablissements de type hospitalier : Nb d’hôpitaux et d'établissements thermaux.

\subsection{Enjeux économiques}

Six indicateurs ont été étudiés pour les enjeux économiques :

- Activités économiques : Nb d'établissements présents en secteur vulnérable ;

- Emploi : Effectifs salariés présents en secteur vulnérable ;

- Bâti : Nb de bâtiments de plus de $20 \mathrm{~m}^{2}$, sans distinction de fonction ;

- Réseau routier : Mètres linéaires de voies de communications Iaire et IIaire ;

- Réseau ferré : Mètres linéaires de voies ferrées ;

- Conduites de matières premières : Mètres linéaires de conduite ou tapis roulant. 


\section{Thème 7 - Risques côtiers}

\subsection{Enjeux liés à l'environnement}

De par les préconisations faites par les services de l'Etat ce sont 4 types d'indicateurs qui ont été étudiés. Les installations nucléaires de base et les centrales nucléaires n’y figurent pas car aucune de ces infrastructures n'est présente sur le territoire étudié :

- Réservoirs d'eau et stations de pompage : Nb de réservoirs et stations de pompage ;

- Stations de Traitement des Eaux Usées (STEU) : Nb de STEU de capacité supérieure à 200 Equivalents Habitants ;

- Installations Classées pour la Protection de l'Environnement (ICPE) : Nb d'ICPE soumises à autorisation ;

- Etablissements SEVESO seuil haut : Nb d'établissements SEVESO « seuil haut ».

\section{Attribution d'une valeur de vulnérabilité aux différents enjeux}

Etant donné les différentes catégories d'enjeux étudiées ce sont plus de 94000 enjeux surfaciques qui ont été testés et 3641 kilomètres de réseaux ferrés, routiers et de conduites de matières premières. C'est un territoire sur lequel la population dépasse les 202000 individus (INSEE, 2009). La figure 1 présente les étapes ayant permis l'attribution d'une valeur de vulnérabilité globale à chacun des 18 types d'enjeux.

La définition des éléments pris en compte pour l'évaluation de la vulnérabilité à l'érosion d'une part, et de la vulnérabilité à la submersion marine et aux autres types d'inondation d'autre part, a permis de générer une couche de vulnérabilité globale. Intégrant les informations liées à l'érosion et aux aléas inondation, cette couche est constituée de pixels de 5 mètres de côté auxquels ont été attribués une valeur de vulnérabilité unique. Elle couvre près de 66123 hectares. Par traitement sous logiciel SIG, les couches d'enjeux ont été superposées à la couche de vulnérabilité globale.

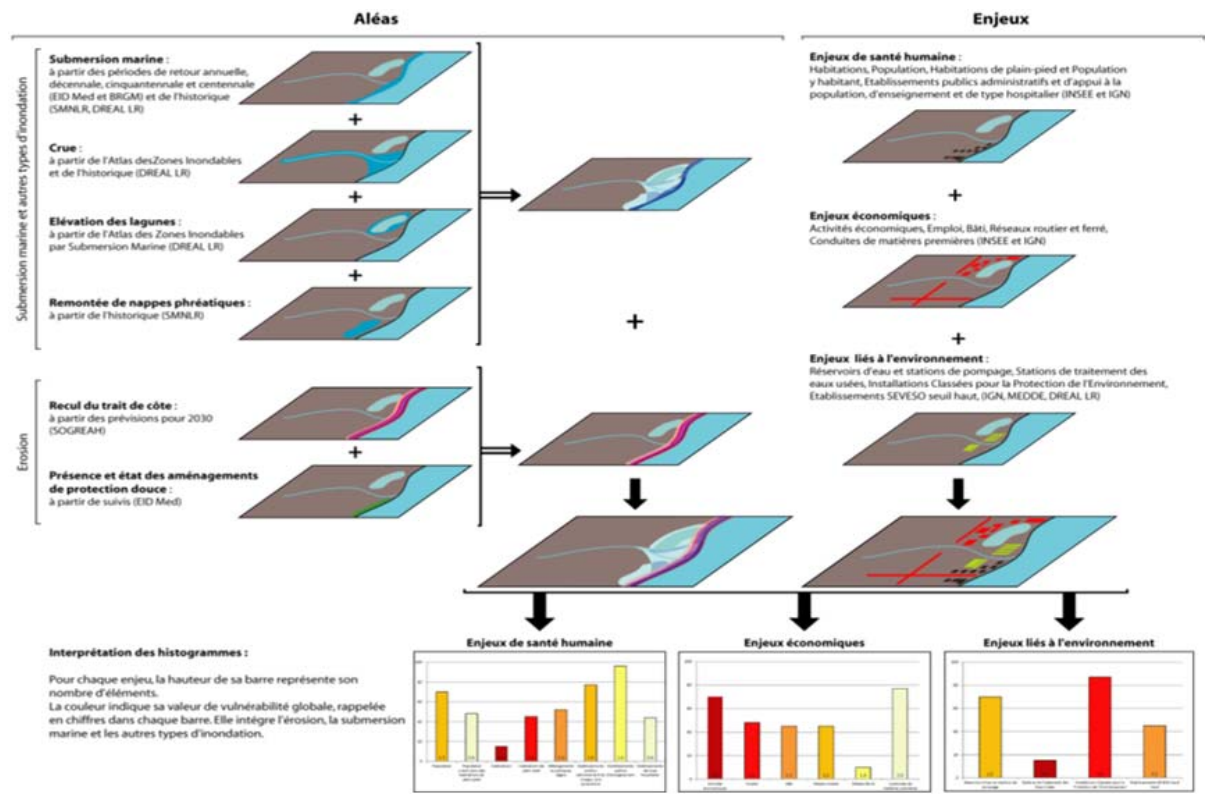

Figure 1. Attribution d'une valeur de vulnérabilité globale aux différents enjeux. 


\section{XIII ${ }^{\text {èmes }}$ Journées Nationales Génie Côtier - Génie Civil \\ Dunkerque, 2-4 juillet 2014}

\section{Présentation des résultats au moyen d'un atlas}

L'ensemble des résultats a ensuite été traité par secteur d'étude. Pour chacun des 38 secteurs, le nombre d'enjeux présent par catégorie a été obtenu ainsi qu'une note de vulnérabilité. Les résultats ont été présentés sous forme de cartes accompagnées d'histogrammes (figure 2).
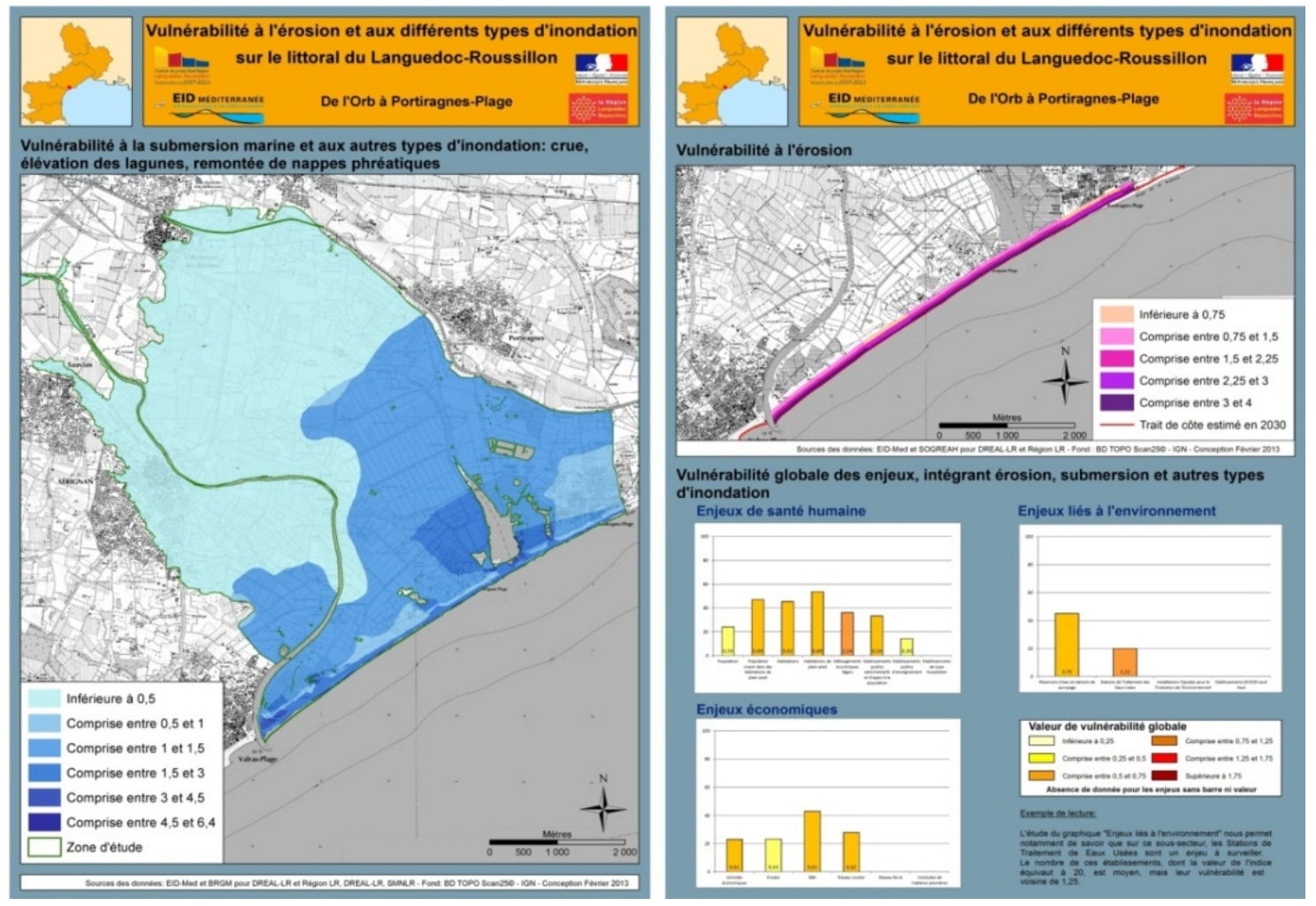

Figure 2. Représentation des résultats pour un secteur d'étude.

\section{Conclusions}

Sur le plan méthodologique, cette étude est apparue intéressante par l'ensemble des paramètres pris en compte, et notamment pour les aléas. Il s'agissait dans ce sens d'une étude multi-aléas et pour laquelle plusieurs périodes de retour ont été mobilisées pour la submersion marine. Ces éléments théoriques ont en outre été précisés de manière sectorielle par un historique des évènements passés sur 30 ans. Cette approche a dans ce sens paru pertinente puisqu'elle a été citée dans le récent guide méthodologique sur les PPR Littoraux (MEDDE, 2013).

L’interprétation des résultats a permis de déterminer les secteurs sur lesquels se produiraient le plus de dommages de façon globale, et les secteurs sur lesquels se produiraient les dommages les plus importants pour des catégories d'enjeux en particulier. Ces deux types d'information, qui se recoupaient sur les secteurs prioritaires, ont permis aux décideurs la définition de grandes orientations de gestion par 
comparaison entre secteurs. Des zones particulièrement exposées ont pu en outre être identifiées au sein de chacun des 38 secteurs.

\section{Remerciements}

Cette étude a été effectuée à partir de financements de l'Etat, de la DREAL LanguedocRoussillon et de la Région Languedoc-Roussillon.

\section{Références bibliographiques}

BRUNEL C. (2013). Définition des limites des cellules de l'atlas des bilans sédimentaires. Rapport d'études, CEFREM, Université de Perpignan Via Domitia.

BRGM (2011). Evaluation des submersions marines lors des tempêtes en LanguedocRoussillon - Validation de la méthodologie. septembre 2011.

DREAL LR -Languedoc-Roussillon- (2009). Atlas numérique des zones inondables par submersion marine - Littoral sableux du LR - Elaboration d'un SIG. janvier 2009.

DREAL LR -Languedoc-Roussillon- (2010). Atlas des zones inondables - SIG Base de données numériques géographiques - Notice. avril 2010.

DREAL LR -Languedoc-Roussillon- (2011). ICPE. février 2011

DREAL LR -Languedoc-Roussillon- (2012). Guide régional d'élaboration des Plans de Prévention des Risques Littoraux Languedoc-Roussillon. novembre 2012.

EID -Entente Interdépartementale pour la Démoustication du littoral Méditerranéen(2011). Annexe Cartographique- Représentation des résultats de l'évaluation des aménagements de protection douce. février 2011.

IGN (2011). BD TOPO® Version 2 - Descriptif de contenu. janvier 2011.

INSEE (2009). RFL 2009 Densité de population en LR. Disponible en ligne sur :

http://www.insee.fr/fr/themes/detail.asp?reg_id=0\&ref_id=donnees-carroyees\&page=donnees-detaillees/donnees-

carroyees/r91.htm

INSEE (2011). Nombre d'établissement et effectifs au 31 décembre 2010. Disponible en ligne sur : http://www.insee.fr/fr/themes/detail.asp?reg_id=99\&ref_id=etab-eff-sal-par-sates

LAPEYRE J. (2004). Mise en place de l'atlas cartographique des zones inondables par submersion marine du L-R. Mémoire de Master, Université de Perpignan.

MEDDE -Ministère de l'écologie, du développement durable, et de l'énergie- (2009). Prim.net - Bouquet prévention risques majeurs. Glossaire risques majeurs. Disponible en ligne sur : http://www.risquesmajeurs.fr/glossaire-risques-majeurs

MEDDE -Ministère de l'écologie, du développement durable, et de l'énergie- (2012). Portail d'information sur l'assainissement communal. Disponible en ligne sur: http://assainissement.developpement-durable.gouv.fr/services.php MEDDE -Ministère de l'écologie, du développement durable, et de l'énergie- (2013). Guide méthodologique : PPR littoraux. Décembre 2013.

SOGREAH (2011). Actualisation de l'aléa érosion en Languedoc-Roussillon - Rapport provisoire. juillet 2011. 